\title{
ОСТАТОЧНАЯ НАМАГНИЧЕННОСТЬ И ВНЕШНЕЕ МАГНИТНОЕ ПОЛЕ ПРЯМОШОВНЫХ СТАЛЬНЫХ ТРУБ, КАК ОБЪЕКТА ИНЖЕНЕРНЫХ ИЗЫСКАНИЙ
}

\author{
В. Н. Глазнев ${ }^{1}$, В. С. Стариков ${ }^{2}$ \\ ${ }^{1}$ Воронежский государственный университет \\ ${ }^{2}$ АО Предприятие подводно-технических работ «Пётр», г. Воронеж \\ Поступила в редакцию 15 августа 2018 г.
}

\begin{abstract}
Аннотация: рассматривается результаты лабораторных измерений остаточной намагниченности прямошовных стальных труб большого диаметра. Установлена преобладающая ориентировка вектора остаточной намагниченности материала в направлении продольной оси трубы. Выявлена четкая связь остаточной намагниченности с техническими условиями изготовления прямошовной трубы. На основе полученных результатов предложена общая модель источника магнитных аномалий, обусловленных изучаемым объектом. Рассматриваются результаты решения прямой задачи магнитометрии от прямошовной трубы для наиболее практически значимых случаев: коллинеарного и антипараллельного направления векторов элементов составной трубы. Результаты измерений можно использовать для анализа технического состояния трубопроводов на основе прямошовных стальных труб по данным детальной магнитной съёмки.
\end{abstract}

Ключевые слова: стальная труба, остаточная намагниченность, магнитные аномалии.

\section{REMANENCE MAGNETIZATION AND EXTERNAL MAGNETIC FIELD OF THE DIRECT-SEAM STEEL PIPES AS AN OBJECT OF ENGINEERING SURVEYS}

\begin{abstract}
: discusses the results of laboratory measurements of residual magnetization direct-seam large diameter steel pipes. Installed the predominant orientation of the vector of residual magnetization of a material in the direction of the longitudinal axis of the tube. Identified a clear link residual magnetization with the technical conditions of manufacture direct-seam pipes. On the basis of the results obtained offered total source model of magnetic anomalies arising from the studied object. The results of the decision are considered direct task of direct-seam pipes for magnetometry most practically significant cases: collinear and antiparallel the direction vectors of elements in composite pipes. The measurement results can be used to assess the technical condition of the pipelines on the basis of direct-seam steel pipe according to detailed magnetic survey data.
\end{abstract}

Key words: steel pipes, remanence magnetization, magnetic anomalies.

\section{Введение}

В настоящее время методы детальной магниторазведки находят широкое применение в решении инженерных задач с целью обнаружения различных искусственных объектов и артефактов в приповерхностной части изучаемой среды. Особое место в таких исследованиях занимают проблемы изучения подземных трубопроводных систем по данным высокоточных магнитных съёмок. Задачами таких съёмок являются: локализация типовых трубопроводных сооружений; определение их пространственного положения и, при благоприятных условиях, диагностирование эксплуатационного состояния трубопроводов, залегающих на глубинах в несколько метров. Дистанционные магнитные исследования трубопро- водов выполняются в различных вариантах: измерений модуля индукции магнитного поля, градиентов модуля индукции и векторных измерений компонент индукции поля [1-10 и др.]. В результате интерпретации материалов таких съёмок оценивается пространственное положение объектов (стальных труб), которое обусловлено методикой и точностью съёмки, а также наличием техногенных помех в поле [1114]. Контроль интерпретационных построений, как правило, осуществляется на основе решения прямой задачи магнитометрии [15-17], опираясь на априорные сведения о магнитных свойствах материала труб $[7,18-20]$ и изменении этих свойств в процессе монтажа и эксплуатации трубопровода $[9,10,13,21]$. 
Как явствует из приведённого краткого списка публикаций, применение методов магниторазведки для дистанционного изучения стальных трубопроводов интенсивно развивается и используется на практике, как в России, так и за рубежом. При этом задачи интерпретации и моделирования магнитных аномалий от стальных трубопроводных систем требуют более детального рассмотрения специфики пространственного распределения намагниченности в изучаемых объектах. Примером такого изучения являются результаты экспериментального исследования магнитной восприимчивости материала прямошовных стальных труб [20], которые демонстрируют неоднородность восприимчивости, как по длине, так и по окружности трубы и зависимость указанного параметра от технического состояния трубы. Наряду с магнитной восприимчивостью, большую роль в формировании магнитных аномалий от трубопроводных систем оказывает и остаточная намагниченность материала трубы $[18,19]$.

В трубопроводных системах, предназначенных для транспортировки нефти и газа, особенно часто используются прямошовные стальные трубы, специфика изготовления которых заключается в горячей прокатке стального листа, служащего заготовкой для трубы, и последующего формирования готового трубного изделия с продольным сварным швом [22; 23]. Роль температуры в технологии изготовления прямошовной трубы очевидна и поэтому представляется интересным экспериментально исследовать величину остаточной намагниченности такой трубы. В итоге эти данные позволят более корректно выполнять пространственную локацию трубопроводов и обеспечивать более достоверную диагностику их технического состояния.

\section{Методика исследований}

Инструментом изучения магнитных свойств прямошовных стальных труб служил лабораторный астатический магнитометр МА-21 (№ 682), используемый для измерений магнитных свойств пород и руд [24] с заметным содержанием ферромагнетиков. Указанный прибор обладает достаточным диапазоном измерений для работы с образцами стали и приемлемой точностью измерений.

Объектами наших исследований стали прямошовные стальные трубы большого диаметра, применяемые в нефтегазовой отрасли. Были изучены стандартные трубы диаметром 530 мм и толщиной стенки 8 мм, которые не подвергались внешним воздействиям и длительное время находились в складских помещениях, сохраняя свои функциональные особенности. Для изготовления образцов, предназначенных для измерений остаточной намагниченности, от тела трубы с помощью абразивного инструмента был отрезан кольцевой фрагмент шириной $5 \mathrm{~cm}$, отстоящий от края трубы примерно на 80 см. С целью равномерного покрытия этого сегмента образцами, на круговом сечении трубы с шагом $30^{\circ}$ задавались сектора, отсчи- тываемые от продольного сварного шва трубы по часовой стрелке (рис. 1). Образец, представляющий соответствующий сектор, с помощью абразивного инструмента отделялся от кольцевого фрагмента трубы.

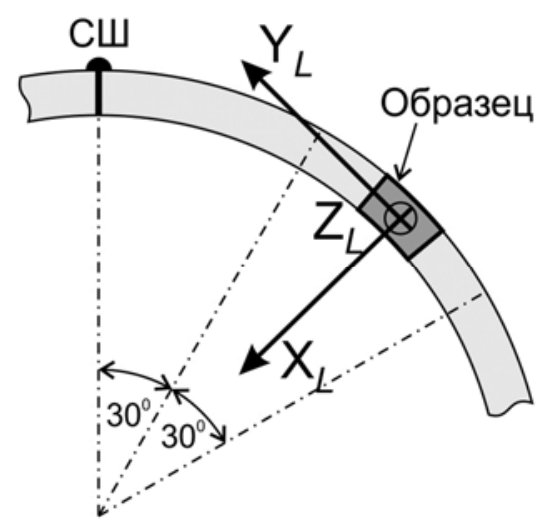

Pис. 1. Пример расположения образца на секторе фрагмента трубы и локальная правая система координат для данного образца: ось $\mathrm{X}_{L}$ - по радиусу трубы, ось $\mathrm{Y}_{L}-$ по касательной к окружности трубы, ось $Z_{L}$ - по простиранию продольного сварного шва (СШ) трубы.

Совокупное количество исследованных сегментов на круговом сечении трубы равнялось двенадцати, что достаточно полно представляет магнитные характеристики всего изделия по окружности трубы, а размеры каждого образца, представляющего по форме слегка изогнутую пластину, составляли примерно 5 x 5 х 0,8 см. Отметим, что все операции по изготовлению образцов выполнялись только с помощью абразивного инструмента и при интенсивном охлаждении водой зоны распила, что исключает возможные изменения магнитных свойств под воздействием нагрева разрезаемой трубы.

Согласно инструкции к прибору МА-21, измерения для каждого из подготовленных образцов тела трубы выполнялись в «равноудалённом положении» при четырёх пространственных ориентировках контейнера с образцом. Такая методика позволяет достаточно надёжно рассчитывать величину магнитного момента образца, по соответствующему направлению локальной системы прямоугольных координат, ориентировка которой показана на рис. 1. Отметим, что принятая ориентировка образцов в процессе измерений на астатическом магнитометре МА-21, была одинаковой для всех сегментов по окружности исследованной трубы. Работа с астатическим магнитометром производилась в лабораторных условиях, вне зоны воздействия помехообразующих электротехнических систем и магнитных объектов, в течение короткого интервала времени, так чтобы минимизировать изменение характеристик магниточувствительной системы прибора. Контроль точности работы астатического магнитометра выполнялся на эталонном ферромагнитном объекте из стального сплава с известными свойствами в форме пластины, геометрически соизмеримой с изучаемыми образцами. 
Компоненты вектора остаточной намагниченности каждого образца рассчитывались по определению [25] делением вычисленных величин магнитных моментов, для соответствующих координат, на объём изучаемого образца. При этом объём образца и его плотность, необходимая для контроля однородности материала стальной трубы, определялась гидростатическим взвешиванием [25], с использованием лабораторных электронных весов НСВ1502.

\section{Результаты измерений}

В результате проведённых измерений и последующей обработки данных была получена сводная таблица данных (табл. 1) по величинам составляющих вектора остаточного намагничения $\mathrm{Jx}_{L}, \mathrm{Jy}_{L}, \mathrm{Jz}_{L}$ в локальных системах координат (рис. 1) для каждого из исследованных образцов. Таблица даёт общее представление о существенной неоднородности распределения намагниченности материала по окружности прямо- шовной трубы, с диапазоном вариаций величин намагничения примерно от -200 А/м до 13000 А/м. Максимальные по модулю значения намагничения, как и следовало ожидать, соответствуют ориентировке длинных осей пластинок образцов в направлении $Y_{L}$ и $Z_{L}$, а величины компоненты остаточного намагничения по оси $\mathrm{X}_{L}$, ортогональной плоскости пластинок образцов, имеют значения примерно на один-два порядка меньше. Отметим, что величины компонент остаточного намагничения, приведённые в таблице, не учитывают влияния размагничивающего фактора, который, согласно оценкам [15, 19], для наших образцов в форме пластинки может достигать 20-30\%. Тем не менее, рассчитанные значения модуля вектора остаточного намагничения Jrem (табл. 1) вполне сопоставимы с результатами измерений на образцах для других стальных труб $[18,19]$ и общими сведениями об остаточном намагничении материала стальных труб большого диаметра [7].

Результаты измерений намагничения образцов стальной трубы

Таблица 1

\begin{tabular}{|c|c|c|c|c|c|}
\hline $\begin{array}{c}\text { Сектор } \\
\text { (градусы) }\end{array}$ & $\begin{array}{c}\mathrm{Jx}_{L} \\
(\mathrm{~A} / \mathrm{M})\end{array}$ & $\begin{array}{c}\mathrm{Jy}_{L} \\
(\mathrm{~A} / \mathrm{M})\end{array}$ & $\begin{array}{c}\mathrm{J}_{L} \\
(\mathrm{~A} / \mathrm{M})\end{array}$ & $\begin{array}{l}\text { Jrem } \\
(\mathrm{A} / \mathrm{M})\end{array}$ & $\begin{array}{c}\text { Jind } \\
(\mathrm{A} / \mathrm{M})\end{array}$ \\
\hline 15 & 34 & 6511 & 8723 & 10885 & 2717 \\
\hline 45 & 274 & 7070 & 11492 & 13495 & 3297 \\
\hline 75 & 142 & 4288 & 13033 & 13721 & 3130 \\
\hline 105 & 81 & 5562 & 12109 & 13325 & 3327 \\
\hline 135 & -29 & 3671 & 11024 & 11619 & 2937 \\
\hline 165 & 131 & 5395 & 8550 & 10110 & 2499 \\
\hline 195 & -52 & 5096 & 7829 & 9342 & 2552 \\
\hline 225 & 328 & 6541 & 8566 & 10783 & 2721 \\
\hline 255 & 215 & 5634 & 9934 & 11422 & 2917 \\
\hline 285 & 120 & 7813 & 7701 & 10971 & 2951 \\
\hline 315 & -194 & 7219 & 6278 & 9569 & 2820 \\
\hline 345 & -27 & 7653 & 5728 & 9559 & 2592 \\
\hline
\end{tabular}

Примечания: $J x_{L}, J y_{L}, J z_{L}$ - компоненты векторов остаточной намагниченности; Jrem - модуль вектора остаточного намагничения; Jind - модуль вектора индуктивного намагничения

Примечательной особенностью в распределении намагничения по плоскости ортогонального сечения трубы является значимое уменьшение величины компоненты $\mathrm{Jz}_{L}$ и возрастание величины компоненты $\mathrm{Jy}_{L}$ вблизи продольного сварного шва трубы (рис. 2). Эффекты такого рода хорошо известны в технологии сварки труб большого диаметра $[26,27]$ и связаны с воздействием мощного поля сварной электрической дуги на ферромагнитный материал тела трубы, а также с термическим перемагничиванием в зоне сварного шва.

Ещё одним следствием из приведённых результатов измерений является преобладание величины модуля остаточного намагничения Jrem по сравнению с величиной модуля индуктивного намагничения Jind. Как явствует их наших данных (табл. 1), величина индуктивного намагничения трубы примерно в тричетыре раза меньше, чем остаточное намагничение. При этом сама величина индуктивного намагничения хорошо соответствует ранее полученным дан- ным [20] по магнитной восприимчивости материала стальных прямошовных труб большого диаметра.

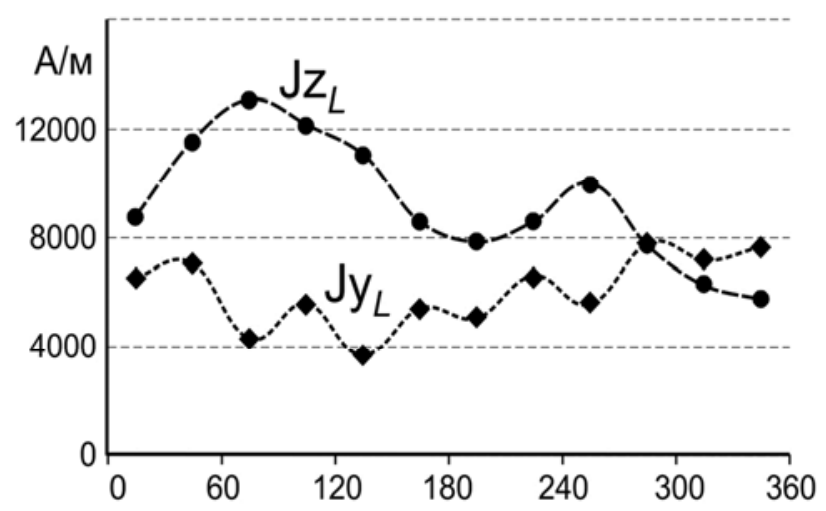

Puc. 2. Величины остаточного намагничения $\mathrm{Jy}_{L}$ и $\mathrm{Jz}_{L}$ для образцов из секторов фрагмента трубы. Горизонтальная ось на графике - углы в градусах по образующей кругового сечения трубы от положения СШ. 
Анализ экспериментальных данных

Приведённые выше результаты натурных измерений остаточной намагниченности на образцах материала реальной прямошовной стальной трубы большого диаметра позволяют обратиться к вопросу о пространственной ориентировке вектора намагничения такого объекта в целом. Далее в наших построениях будем пренебрегать малой величиной радиальной компоненты остаточной намагниченности образцов трубы, ориентированной по оси $\mathrm{X}_{L}$ (рис. 1 ), и рассматривать вклад только наиболее интенсивных компонент $\mathrm{Jy}_{L}$ и $\mathrm{Jz}_{L}$ (табл. 1), лежащих в плоскости образующей окружности прямошовной трубы. По величинам намагничения для двух этих компонент можно приближённо вычислить угол $\alpha$ между горизонтальным вектором намагничения $\mathrm{Jy}_{L}$, лежащим в плоскости образца, и вектором $\mathrm{Jz}_{L}$, ориентированным параллельно продольному сварному шву трубы, согласно соотношению:

$$
\alpha=\operatorname{arctg}\left(\mathrm{Jy}_{\mathrm{L}} / \mathrm{Jz}_{\mathrm{L}}\right)
$$

Рассчитанные значения углов, показанные на рис. 3 для всех образцов, демонстрируют примерно закономерное отклонение векторов горизонтального намагничения от направления оси $Z_{L}$. Величины углов $\alpha$, для большинства образцов материала трубы, лежат в диапазоне от 18 до 37 градусов и только в краевой зоне вблизи сварного шва увеличиваются до 45-55 градусов.

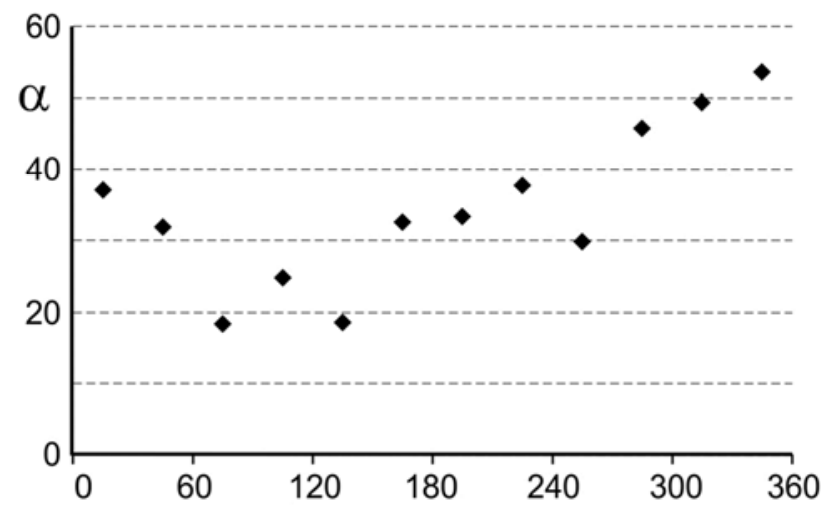

Puc. 3. Значения угла $\alpha$ (в градусах) между полным горизонтальным вектором остаточного намагничения образца и вектором $\mathrm{JZ}_{L}$. Горизонтальная ось на графике - углы в градусах по образующей кругового сечения трубы от положения СШ.

Выявленная примерная однородность направлений векторов полного горизонтального намагничения образцов для всех секторов трубы, в системе локальных координат этих образцов, позволят обратиться к расчёту векторов намагничений в системе координат всего изделия, которую в дальнейшем будем называть глобальной. В качестве таковой возьмём систему, связанную с ориентировкой продольного сварного шва трубы, а именно: ось $Z_{P}$ - по простиранию радиальной оси трубы, ось $\mathrm{X}_{P}$ - лежит в плоскости сечения трубы и направлена от её центра к сварному шву трубы, ось
$\mathrm{Y}_{P}$ - лежит в плоскости сечения трубы, образуя левую систему координат. Связь между указанными системами координат всего изделия (трубы) и локальными системами координат образцов сегментов трубы определяется аффинными преобразованиями:

$$
\begin{gathered}
\mathrm{X}_{\mathrm{p}}=\mathrm{X}_{\mathrm{L}} \cdot \cos \varphi+\mathrm{Y}_{\mathrm{L}} \cdot \sin \varphi, \\
\mathrm{Y}_{\mathrm{p}}=-\mathrm{X}_{\mathrm{L}} \cdot \sin \varphi+\mathrm{Y}_{\mathrm{L}} \cdot \cos \varphi, \\
\mathrm{Z}_{\mathrm{p}}=\mathrm{Z}_{\mathrm{L}},
\end{gathered}
$$

где $\varphi$ - угол по круговому сечению трубы между центром соответствующего образца и сварным швом.

Полученные таким образом значения намагниченности $\mathrm{Jx}_{P}$ и Ју в глобальной системе координат показаны на рис. 4. Как явствует из рисунка, амплитуды этих компонент хорошо отвечают гармоническим зависимостям от угла $\varphi$, аппроксимация которых даётся соотношениями:

$$
\begin{array}{r}
\mathrm{J}_{\mathrm{p}}=\mathrm{J} \varphi_{\mathrm{P}} \cdot \sin \varphi, \\
\mathrm{Jy}_{\mathrm{p}}=\mathrm{J} \varphi_{\mathrm{P}} \cdot \cos \varphi,
\end{array}
$$

где $\mathrm{J} \varphi_{P}=6050(\mathrm{~A} / \mathrm{M})$ - амплитуда усреднённой тангенциальной компоненты остаточного намагничения, направленной по касательной к круговому сечению трубы в цилиндрической глобальной системе координат. Напомним, что значение намагничения по продольной оси трубы остаётся неизменными, поскольку $\mathrm{J}_{P}=\mathrm{Jz}_{L}$ (рис.2).

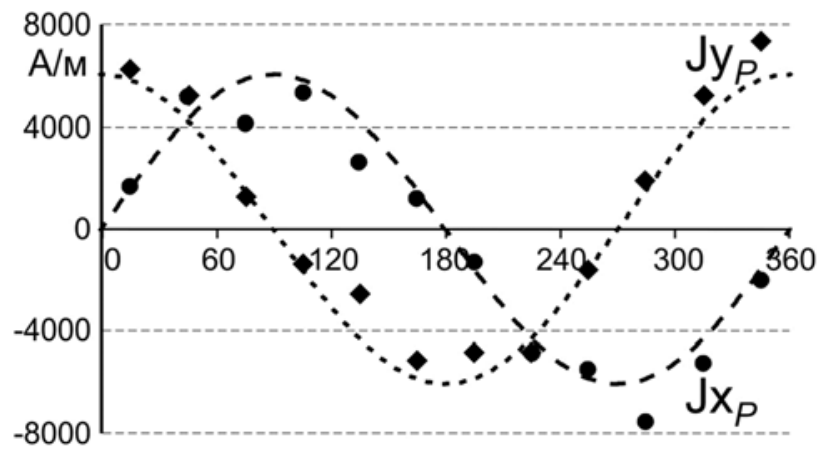

Puc. 4. Величины остаточного намагничения $\mathrm{Jx}_{P}$ и $\mathrm{Jy}_{P}$ образцов материала трубы в глобальной системе координат изделия. Пунктирные линии показывают аппроксимацию зависимостей согласно выражению (3). Горизонтальная ось на графике - углы в градусах по образующей кругового сечения трубы от положения СШ.

Приведённые результаты расчётов величин $\mathrm{Jx}_{P}, \mathrm{Jy}_{P}$ и $\mathrm{J}_{P}$ допускают простую трактовку пространственного положения вектора остаточной намагниченности всего изделия - данный вектор, образованный тангенциальной $\mathrm{J} \varphi_{P}$ и продольной $\mathrm{Jz}_{P}$ компонентами, лежит практически в плоскости стального листа, представляющего заготовку будущей трубы. Известно [23], что стандартные размеры формовочного листа для рассматриваемого типа труб большого диаметра со- 
ставляют примерно 1,7 м на 12 м, а толщина листа 8 мм. Таким образом, остаточное намагничение материала трубы формируется в плоскости тонкого формовочного листа, что отвечает теоретическим моделям намагничения для тонкого листа конечного размера [28].

Образование остаточной намагниченности материала формовочного листа обусловлено процессом термоостаточного намагничения стали на стадии горячей прокатки формовочного листа при температуре выше точки Кюри, составляющей примерно 670 $720 \mathrm{C}^{\circ}$ для данных сортов стали. В процессе последующего сворачивания листа-заготовки вектор остаточного намагничения не претерпевает значительных изменений, поскольку материал трубы уже охлаждён ниже температуры Кюри [23, 29]. Таким образом, термоостаточная намагниченность материала трубы формируется на стадии прокатки листа, а ориентировка вектора остаточного намагничения определяется, в основном, размерами и первичной формой листазаготовки.

Положение векторов остаточного намагничения материала нашей листовой заготовки показано на рис. 5. Здесь величина $\mathrm{Jh}_{P}$ представляет модуль вектора остаточного намагничения в плоскости поперечного сечения трубы, вычисленный для каждого образца на основании очевидных соотношений:

$$
J h_{P}=\sqrt{\left(J x_{P}\right)^{2}+\left(J y_{P}\right)^{2}}=\sqrt{\left(J x_{L}\right)^{2}+\left(J y_{L}\right)^{2}},
$$

а угол отклонения полного вектора остаточного намагничения от направления вектора $\mathrm{Jz}_{P}$ определяется приближённым соотношением (1). Разумеется, приведённый рисунок даёт не полного описания остаточного намагничения всего изделия, поскольку величины компонент намагничения достаточно неоднородны по листу заготовки (табл. 1) и, кроме того, количество изученных образцов было ограничено единственным участком трубы по её длине. Тем не менее, полученные данные позволяют говорить о характере ориентировки вектора остаточного намагничения стальной прямошовной трубы - этот вектор ориентирован строго в плоскости листа-заготовки. Для конкретной изученной по одному элементу сечения трубы средние значения продольной и тангенциальной компонент остаточного намагничения составляют, соответственно, $\mathrm{Jz}_{P}=9250 \mathrm{~A} / \mathrm{M}$ и $\mathrm{Jh}_{P}=$ 6050 A/M, а среднее значение модуля вектора остаточного намагничения материала трубы Jrem = $11050 \mathrm{~A} / \mathrm{M}$.

Приведённые результаты анализа экспериментальных данных для образцов материала трубы показывают, что прямошовная стальная труба, по своим магнитным свойствам, является достаточно сложным трёхмерным объектом. Очевидно, что расчёт магнитного поля от такого объекта на основе простейших аппроксимаций тела трубы в виде прямолинейного ограниченного отрезка тонкого горизонтального цилиндра или эллипсоид вращения [7,
$16,17]$, обладающих одноосным однородным намагничением, - не представляется корректным. Наши экспериментальные данные позволяют обратиться к созданию содержательной математической модели тела, аппроксимирующего стальную прямошовную трубу.

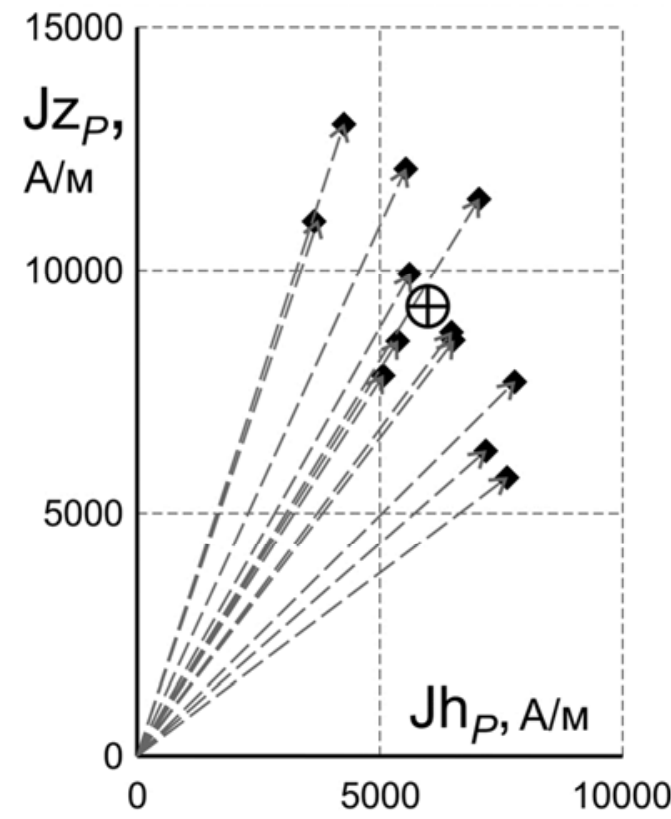

Puc. 5. Положение векторов остаточного намагничения (серые стрелки) и значения компонент $\mathrm{Jz}_{P}$ и $\mathrm{Jh}_{P}$ образцов материала трубы (чёрные ромбы) на развёртке листа трубной заготовки. Показано среднее значение величины компонент остаточного намагничения (окружность с перекрестьем) для всего набора данных.

\section{Магнитное поле прямошовной трубы}

Рассмотрим задачу вычисления магнитного поля от нашего модельного объекта на основе аппроксимационного подхода, который позволяет эффективно решать задачи для сложных объектов [30]. В качестве базового аппроксимирующего элемента модели возьмём тонкий горизонтальный ограниченный по простиранию цилиндр с произвольным направлением вектора намагничения. Аналитические выражения компонент индукции магнитного поля от этого объекта можно вычислить интегрированием компонент индукции поля произвольно намагниченного магнитного диполя [24] по одной из осей координат, например Y, полагая, что сформированный таким образом тонкий цилиндр («нить диполей») ориентирован вдоль этой оси.

Итоговые выражения компонент индукции магнитного поля от элементарного аппроксимационного объекта (записанные в традиционной для магниторазведки координатной системе, где ось $Z$ направлена вниз, ось Y - по простиранию объекта, а ось $\mathrm{X}$ ортогональна двум другим, образуя левую систему координат) имеют вид: 


$$
\begin{aligned}
& d B_{X}(x, y, z)=\frac{\mu_{0}}{4 \pi} d S\left\{\frac{(x-\xi)^{2} J_{X}-(x-\xi)(z-\zeta) J_{Z}}{r^{4}}\left[\frac{\left(y-L_{2}\right)^{3}}{\left[r^{2}+\left(y-L_{2}\right)^{2}\right]^{3 / 2}}-\frac{\left(y-L_{1}\right)^{3}}{\left[r^{2}+\left(y-L_{1}\right)^{2}\right]^{3 / 2}}\right]+\right. \\
& +\frac{3(x-\xi)(z-\zeta) J_{Z}+\left[(z-\zeta)^{2}-2(x-\xi)^{2}\right] J_{X}}{r^{4}}\left[\frac{\left(y-L_{2}\right)}{\left[r^{2}+\left(y-L_{2}\right)^{2}\right]^{1 / 2}}-\frac{\left(y-L_{1}\right)}{\left[r^{2}+\left(y-L_{1}\right)^{2}\right]^{1 / 2}}\right]+ \\
& \left.+(x-\xi) J_{Y}\left[\frac{1}{\left[r^{2}+\left(y-L_{2}\right)^{2}\right]^{3 / 2}}-\frac{1}{\left[r^{2}+\left(y-L_{1}\right)^{2}\right]^{3 / 2}}\right]\right\} \text {, } \\
& d B_{Y}(x, y, z)=\frac{\mu_{0}}{4 \pi} d S\left\{\left[(x-\xi) J_{X}-(z-\zeta) J_{Z}\right]\left[\frac{1}{\left[r^{2}+\left(y-L_{2}\right)^{2}\right]^{3 / 2}}-\frac{1}{\left[r^{2}+\left(y-L_{1}\right)^{2}\right]^{3 / 2}}\right]+\right. \\
& \left.+\frac{J_{Y}}{r^{2}}\left[\frac{\left(y-L_{2}\right)^{3}}{\left[r^{2}+\left(y-L_{2}\right)^{2}\right]^{3 / 2}}-\frac{\left(y-L_{1}\right)^{3}}{\left[r^{2}+\left(y-L_{1}\right)^{2}\right]^{3 / 2}}\right]+\frac{J_{Y}}{r^{2}}\left[\frac{\left(y-L_{2}\right)}{\left[r^{2}+\left(y-L_{2}\right)^{2}\right]^{1 / 2}}-\frac{\left(y-L_{1}\right)}{\left[r^{2}+\left(y-L_{1}\right)^{2}\right]^{1 / 2}}\right]\right\} \text {, } \\
& d B_{Z}(x, y, z)=\frac{\mu_{0}}{4 \pi} d S\left\{\frac{(z-\zeta)^{2} J_{X}-(x-\xi)(z-\zeta) J_{X}}{r^{4}}\left[\frac{\left(y-L_{2}\right)^{3}}{\left[r^{2}+\left(y-L_{2}\right)^{2}\right]^{3 / 2}}-\frac{\left(y-L_{1}\right)^{3}}{\left[r^{2}+\left(y-L_{1}\right)^{2}\right]^{3 / 2}}\right]+\right. \\
& +\frac{3(x-\xi)(z-\zeta) J_{X}+\left[(x-\xi)^{2}-2(z-\zeta)^{2}\right] J_{Z}}{r^{4}}\left[\frac{\left(y-L_{2}\right)}{\left[r^{2}+\left(y-L_{2}\right)^{2}\right]^{1 / 2}}-\frac{\left(y-L_{1}\right)}{\left[r^{2}+\left(y-L_{1}\right)^{2}\right]^{1 / 2}}\right]+ \\
& \left.+(z-\zeta) J_{Y}\left[\frac{1}{\left[r^{2}+\left(y-L_{2}\right)^{2}\right]^{3 / 2}}-\frac{1}{\left[r^{2}+\left(y-L_{1}\right)^{2}\right]^{3 / 2}}\right]\right\} \text {. }
\end{aligned}
$$

Здесь переменные $(x, y, z)$ относятся к точке вычисления поля, а переменные $(\xi, \zeta)$ - к точке пространственного положения источника по осям $\mathrm{X}$ и $\mathrm{Z}$ соответственно. Координаты $L_{1}$ и $L_{2}\left(L_{1}<L_{2}\right)$ обозначают положение краевых точек тонкого цилиндра по оси Y. Величина $r$ в (5)-(7) выражает расстояние между центром нашего тонкого цилиндра и точкой вычисления поля в плоскости X0Z согласно определению:

$$
r=\left[(x-\xi)^{2}+(z-\zeta)^{2}\right]^{1 / 2} .
$$

В приведённых выражениях также обозначены: $\mu_{0}$ - магнитная проницаемость вакуума; $d S$ - площадь поперечного сечения элементарного аппроксимаци- онного элемента (тонкого цилиндра); $J_{x}, J_{y}$ и $J_{z}$ - компоненты вектора намагничения этого элемента по соответствующим осям координат.

Выражения для компонент индукции (5) - (7) могут быть использованы для получения аналитических выражений магнитного поля от нашего объекта (трубы) посредством интегрирования в цилиндрических координатах по радиусу $d r$ и углу $d \varphi$ в системе координат источника. Однако, в конечном виде, поле определяется через эллиптические интегралы, которые не упрощают решение конечной задачи - вычисление компонент индукции на некоторой внешней области. Здесь представляется более разумным численное интегрирование аппроксимационных кон- 
струкций (5) - (7) по координатам всей трубы, с использованием полученных аналитических соотношений для компонент вектора остаточного намагничения в форме (3) и установленных оценок величин продольной $\left(J_{y}=9250 \mathrm{~A} / \mathrm{M}\right)$ и тангенциальной $\left(J_{h}=\right.$ $6050 \mathrm{~A} / \mathrm{м})$ компонент остаточного намагничения. Тогда выражения для компонент индукции магнитного поля, обусловленного полным модельным объектом (трубой), можно записать на основе выражений (5) (7) в форме:

$$
\begin{aligned}
& B_{X}(x, y)=\int_{R_{1}}^{R_{2}} \int_{0}^{2 \pi} d B_{X}(x, y, 0) \cdot r d r d \varphi, \\
& B_{Y}(x, y)=\int_{R_{1}}^{R_{2}} \int_{0}^{2 \pi} d B_{Y}(x, y, 0) \cdot r d r d \varphi, \\
& B_{Z}(x, y)=\int_{R_{1}}^{R_{2}} \int_{0}^{2 \pi} d B_{Z}(x, y, 0) \cdot r d r d \varphi,
\end{aligned}
$$

где $R_{1}$ и $R_{2}$ - внутренний и внешний радиусы трубы, а переменные координаты источника $\xi$ и $\zeta$, фигурирующие в (5) - (7), определены как

$$
\begin{gathered}
\xi=\frac{1}{2}\left(R_{1}+R_{2}\right) \cdot \sin \varphi, \\
\zeta=H_{C}-\frac{1}{2}\left(R_{1}+R_{2}\right) \cdot \cos \varphi,
\end{gathered}
$$

где $H_{c}$ - глубина залегания центра трубы. Кроме того, компоненты вектора остаточного намагничения, фигурирующие в (5) - (7), на основании (3) определим как

$$
\begin{aligned}
& J_{x}=J_{h} \cdot \cos \varphi, \\
& J_{y}=J_{h} \cdot \sin \varphi
\end{aligned}
$$

По приведённым соотношениям (5) - (15) можно численно рассчитать компоненты индукции магнитного поля на плоскости наблюдений $z=0$. В нашем моделировании использовалась система компьютерной математики MathCad15 [31], которая позволяет эффективно вычислять интегралы типа (9)-(11). Точность вычислительной схемы определяется величиной $d S=r \cdot d r \cdot d \varphi$, которая должна гарантировать примерное равенство линейных размеров конечного элемента площади. В нашем случае величина $d \varphi$ принималась равной $1^{\circ}$ дуги по окружности трубы, а $d r=0,008$ м - толщине стенки трубы. Принятые параметры обеспечивают точность вычислений магнитного поля объекта порядка $10^{-5}$ нТл в области максимума аномалии, что было проверено прямыми расчётами при увеличении количества узлов интегрирования.

Поскольку на практике инженерной магнитометрии используются приборы измеряющие модуль вектора магнитной индукции, то при расчётах вычислялся модуль вектора индукции, определяемый как

$$
|B(x, y)|=\left[\left(B_{X}(x, y)\right)^{2}+\left(B_{Y}(x, y)\right)^{2}+\left(B_{Z}(x, y)\right)^{2}\right]^{1 / 2}
$$

Пример вычисления карты модуля вектора индукции магнитного поля, обусловленного одним элементом прямошовной трубы диаметром 530 мм и стандартной длиной 12 м, показан на рис. 6. При расчётах принималась во внимание только остаточная намагниченность материала трубы, которая, как было показано выше, существенно преобладает над индуктивной намагниченностью. Приведённые результаты демонстрируют основные особенности аномального магнитного поля от нашего объекта: над концевыми срезами трубы наблюдаются интенсивные максимумы модуля вектора индукции, а над центральной частью объекта отмечается локальное понижение поля. Эти особенности морфологии аномального поля и распределение его амплитуд хорошо согласуются с реальными данными наблюдений и модельными расчётами ряда авторов [2, 6, 7, 11, 14, 17, 30 и др.].

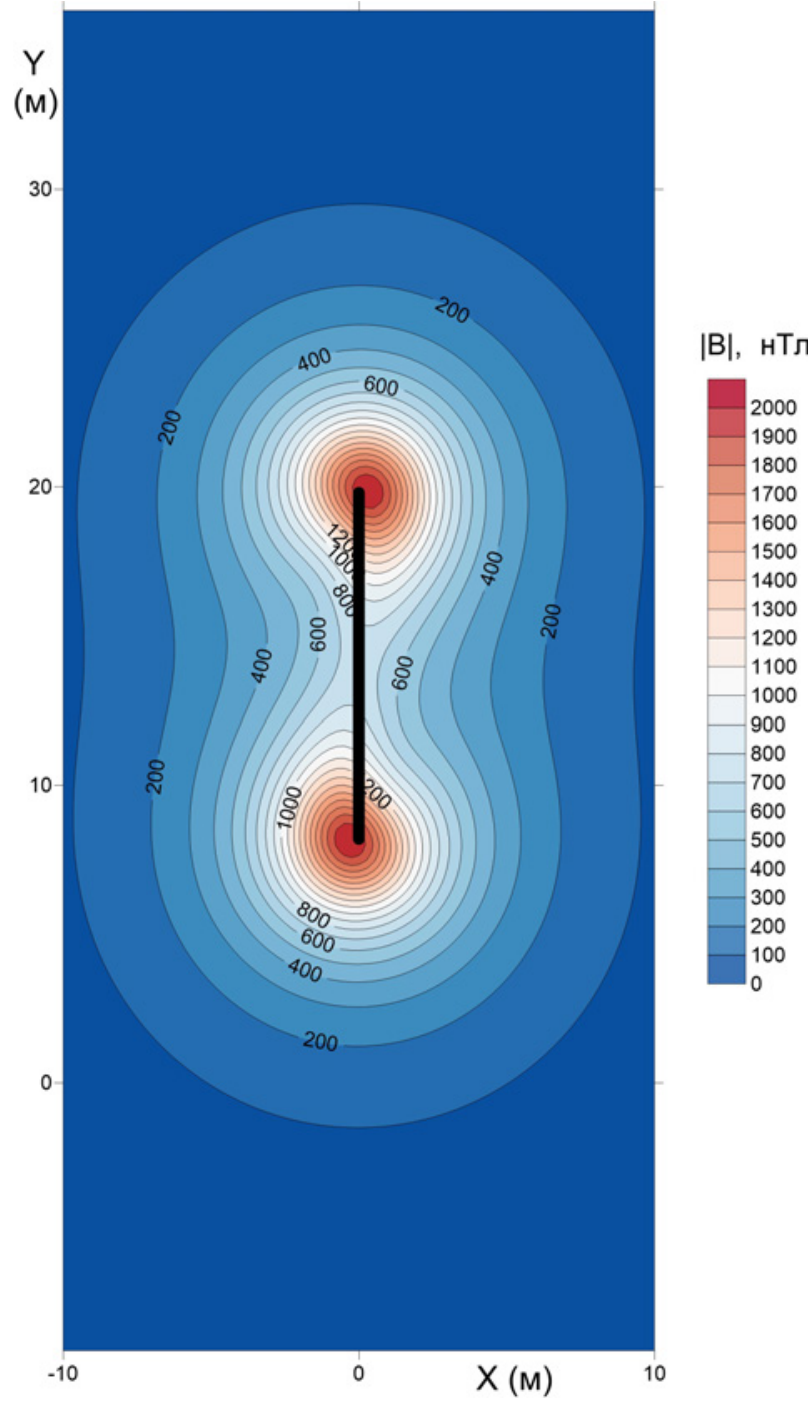

Puc. 6. Карта модуля вектора индукции магнитного поля над стандартной прямошовной трубой длиной 12 м. Плановое положение трубы показано жирной чёрной линией. Глубина залегания центра трубы $H_{\mathrm{c}}=2.5 \mathrm{M}$.

Наибольший интерес при проведении магнитных съёмок над трубопроводными системами вызывает наличие т.н. «чёточных» аномалий магнитного поля, которые представляют последовательность вытянутых вдоль трассы трубопровода локальных экстрему- 
мов поля. Природа таких типичных аномалий объясняется авторами [2, 6, 7, 14 и др.] как результат векторного сложения полей отдельных труб, составляющих трубопровод, и обладающих противоположно направленными векторами остаточного намагничения. Справедливость этого утверждения можно непосредственно проверить в рамках нашей модели расчётов аномального магнитного поля, обусловленного остаточным намагничением прямошовной стальной трубы.

Пример вычисления карт модуля вектора индукции магнитного поля, обусловленного двумя сочленёнными элементами прямошовных труб диаметром 530 мм и длиной 12 м каждая, обладающих одинаковыми величинами остаточного намагничения, показан на рис. 7. Очевидно, что магнитные аномалии нашего составного объекта существенно зависят от взаимного направления компонент векторов остаточного намагничения. В случае параллельности векторов намагничения соседних труб (рис. 7а) аномалия магнитного поля над стыком труб не образуется, что вполне согласуется с общетеоретическими позициями формирования магнитных аномалий от ограниченных по простиранию объектов $[15,24]$. В противоположность этому, при антипараллельности векторов остаточного намагничения (рис. 7б) магнитная аномалия над стыком труб более интенсивна, чем над концевыми частями составной трубы.

Приведённые примеры моделирования магнитных аномалий от составной трубы, показанные на рис. 7, являются весьма упрощёнными, поскольку: намагничение отдельных труб принималось постоянным; не учитывались эффекты изменения намагничения в зоне сварных швов труб; не принималось во внимание индуктивное намагничение составного объекта; не учитывалось дополнительное намагничивание в собственном поле объекта. Указанные факторы могут несколько усложнить картину аномального поля, но принципиально её не изменят, что было проверено на моделях.
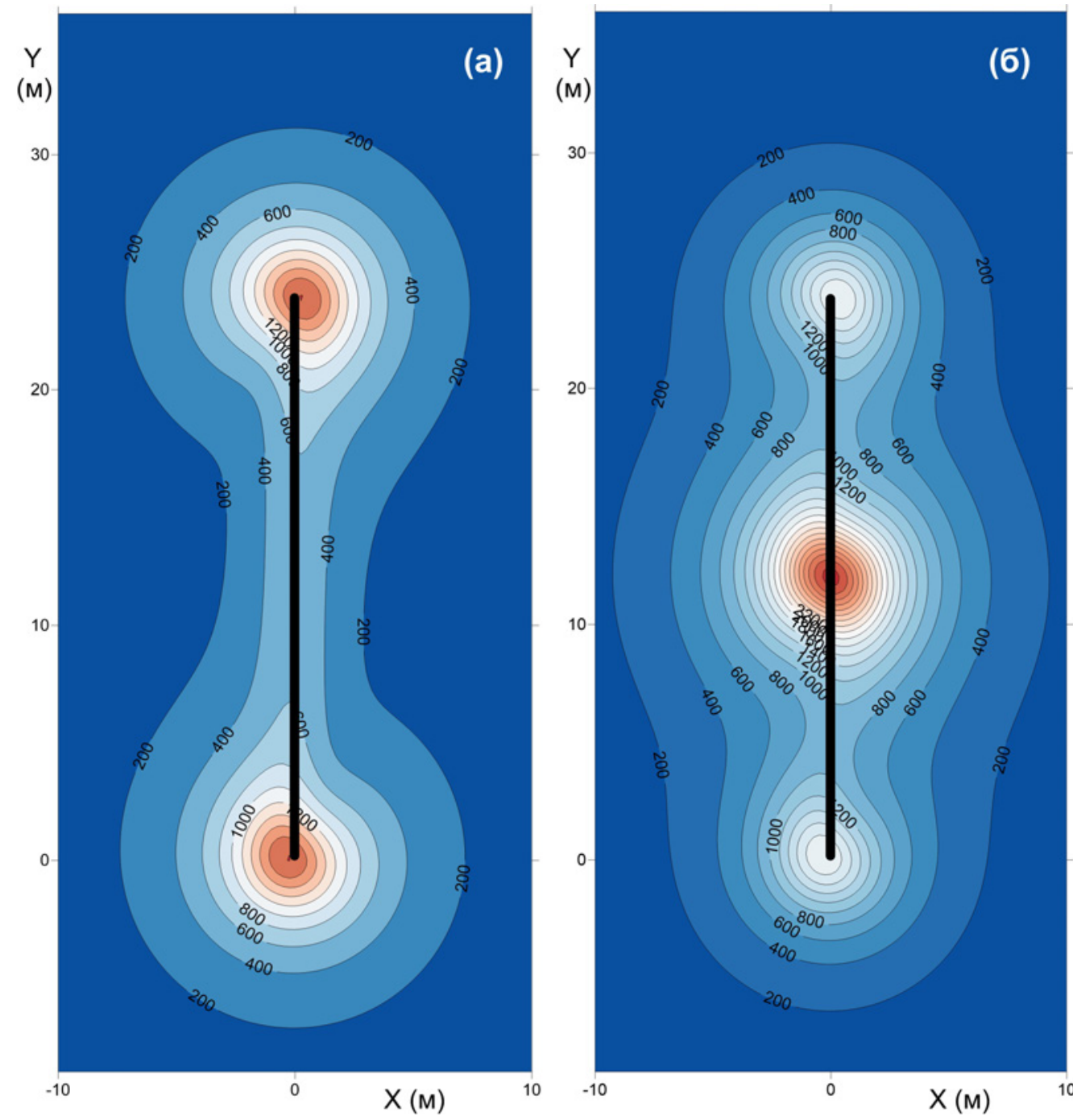

$|\mathrm{B}|$, нТл

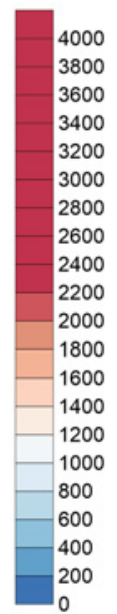

Puc. 7. Карты модуля вектора индукции магнитного поля над составной трубой из двух стандартных прямошовных труб длиной по 12 м каждая: $a$ - компоненты вектора остаточного намагничения труб параллельны; $\sigma$ - компоненты вектора остаточного намагничения труб антипараллельны. Плановое положение составной трубы показано жирной чёрной линией. Глубина залегания центра трубы $H_{\mathrm{c}}=2,5 \mathrm{м}$. 
Таким образом, вычислительные результаты демонстрируют два основных варианта морфологии аномального магнитного поля, которые вполне могут транслироваться на аномальное поле всего трубопровода и подтверждают справедливость тезиса о природе «чёточных» аномалий [2, 6, 7, 14 и др.], формируемых за счёт различия в ориентировке остаточного намагничения отдельных сегментов труб.

\section{Выводы}

Предложенные вниманию читателей результаты экспериментального исследования магнитных свойств прямошовных труб и численного моделирования магнитного поля от такого рода объектов, позволяют сделать следующие выводы:

1. Прямые измерения магнитных свойств материала прямошовной стальной трубы большого диаметра демонстрируют преобладание остаточной компоненты намагничения над индуктивной компонентой.

2. Характерная ориентировка вектора остаточного намагничения прямошовной стальной трубы позволяет утверждать, что термоостаточная намагниченность материала формируется на стадии прокатки листазаготовки трубы.

3. Предложены простые численные методы расчёта компонент индукции внешнего магнитного поля рассматриваемого объекта, основанные на аппроксимационном подходе и опирающиеся на выявленные соотношения для компонент вектора остаточного намагничения материала трубы.

4. Моделирование магнитных аномалий от составной трубы, содержащей несколько отдельных соединённых элементов, демонстрируют два типовых варианта морфологии внешнего аномального магнитного поля над трубопроводом, объясняющих природу «чёточных» аномалий.

Авторы выражают благодарность рецензенту проф. А. С. Долгалю за полезное обсуждение предмета исследований и ряд критических замечаний, которые, несомненно, улучшили содержание статьи.

\section{ЛИТЕРАТУРА}

1. Владов, М. Л. Обзор геофизических методов исследований при решении инженерно-геологических и инженерных задач / М. Л. Владов, А. В. Старовойтов. - М.: ГСД Продакшен. $-1998 .-67 \mathrm{c}$.

2. Геоэкологическое обследование предприятий нефтяной промышленности / Ред.: И. Н. Модин, В. А. Шевнин. - М.: PУCCO. - 1999. - $511 \mathrm{c}$.

3. Манштейн, А. К. Малоглубинная геофизика. (Пособие по спецкурсу) / А. К. Манштейн. - Новосибирск: Изд. НГУ. $-2002 .-135 \mathrm{c}$.

4. Альбанова, E. В. Исследование возможностей оценки технического состояния трубопроводов с помощью магнитных методов / Е. В. Альбанова, Е. И. Крапивский, В. О. Некучаев // Защита окружающей среды в нефтегазовом комплексе, 2005. - № 11. - С. 9-12.

5. Галлямов, И. И. Опыт применения магнитной съёмки на внутрипромысловых трубопроводах как способа технического диагностирования / И. И. Галлямов, А. А. Крылов, А.
И. Галлямов // Тезисы докладов Всерос. научн.-техн. конф. «Современные технологии нефтегазового дела». - Уфа, УГНТУ. - 2007, - С. 46-47.

6. Глазнев, В. Н. Применение методов магнитометрической съёмки для исследования подводных переходов трубопроводов различной протяжённости в условиях мелководья / В. Н. Глазнев, В. С. Стариков // Сборник докладов 5-ой Междун. научн.-прак. конф. и выставки. «Инженерная и рудная геофизика 2009» [Электронный ресурс] DOI: 10.3997/22144609.201403827.

7. Крапивский, Е. И. Дистанционная магнитометрия газонефтепроводов / Е. И. Крапивский, В. О. Некучаев. - Ухта: Изд. УГТУ. - 2011. - 142 с.

8. Любчик, А. Н. Способ дистанционного магнитометрического контроля технического состояния магистральных трубопроводов / А. Н. Любчик // Записки Горного института, 2012. - Т. 195. - С. 268-271.

9. A detection system for pipeline direction based on shielded geomagnetic field / W. Zhao [et al.] // Int. J. of Pressure Vessels and Piping, 2014. - 113. - № 1. - P. 10-14.

10. Huang, $S$. New Technologies in Electromagnetic Nondestructive Testing. Springer Series in Measurement Science and Technology / S. Huang, S. Wang. - Tsinghua University Press. - 2016. - 233 р. [Электронный ресурс] DOI 10.1007/ 978-981-10-0578-7.

11. Гершанок, Л. A. Малоглубинная магниторазведка в условиях промышленных помех / Л. А. Гершанок // Вестник Пермского университета. Сер.: Геология, 2013. - Вып. 1 (18). - C. 34-49.

12. Глазнев, В. Н. Оценка разрешающей способности магнитного градиентометрического метода при решении инженерных геофизических задач: Мат-лы XVI междун. конф. «Структура, свойства, динамика и минерагения литосферы Восточно-Европейской платформы». Под. ред. Н. М. Чернышова, А. А. Маловичко / В. Н. Глазнев, В. С. Стариков. - Воронеж, изд-во. «Научная книга». - 2010. - С. 193-196.

13. Коннов, В. В. Некоторые вопросы оптимизации магнитометрического метода контроля напряжённо-деформированного состояния подземного стального трубопровода / В. В. Коннов // Нефтегазовое дело, 2012. - № 6. - С. 163-176.

14. Инженерная магниторазведка при обнаружении подземных коммуникаций в условиях помех техногенного происхождения / П. Н. Новикова [и др.] // 18-я молодёжная научн. школа по геофизике. Сборник научных материалов. Пермь: ГИ УрО РАН. - 2017. - С. 147-151.

15. Блох, Ю. И. Решение прямых задач гравиразведки и магниторазведки: Учебное пособие / Ю. И. Блох. - М.: изд. МГГА. - 1993. $-79 \mathrm{c}$.

16. Агиней, $P$. В. Моделирование магнитных аномалий при проведении магнитометрического контроля трубопроводов с поверхности грунта / Р. В. Агиней, В. В. Мусонов, С. С. Гуськов // Трубопроводный транспорт, 2013. - №1. - С. 40-44.

17. Загидулин, T. Р. Расчёт магнитного поля стальной трубы конечной протяжённости в постоянном однородном намагничивающем поле / Т. Р. Загидулин // Контроль и диагностика, 2014. - № 6. - С.15-24. [Электронный ресурс] DOI:10.14489/td.2014.06.

18. Ravat, D. Magnetic properties of unrusted steel drums from laboratory and field-magnetic measurements / D. Ravat // Geophysics, 1996. - 61. - P. 1325-1357.

19. Eskola, L. Measurement of magnetic properties of steel sheets / L. Eskola, R. Puranen, H. Soininen // Geophysical Prospecting, 1999. - 47. - P. 593-602.

20. Стариков, В. С. Инженерная магнитометрия при исследовании технического состояния стальных трубопроводов большого диаметра / В. С. Стариков // Вестн. Воронеж. гос. 
ун-та. - Сер.: Геология. - 2016. - №3. - С. 114-118.

21. Magnetic charge and magnetic field distributions in ferromagnetic pipe / H. Xinjing [et al.] // Applied Computational Electromagnetics Society Journal, 2013. - 28. - № 8. - P. 737 746.

22. Николаев, Г. А. Сварные конструкции. Технология изготовления. (Учебное пособие) / Г. А. Николаев, С. А. Куркин, В. А. Винокуров. - М.: Высшая школа. - 1983. - 344 с.

23. Трубы стальные электросварные прямошовные. ГОСТ 10704-91 / В. П. Сокуренко [и др.] - М.: Росстандарт. 2015.

24. Магниторазведка. Справочник геофизика / Под ред. В. Е. Никитского, Ю. С. Глебовского. - М.: Недра. - 1980. $367 \mathrm{c}$.

25. Физические свойства пород и полезных ископаемых. Справочник геофизика / Ред. Н. Б. Дортман. - М.: Недра. 1984. $-455 \mathrm{c}$.

\section{Воронежский государственный университет}

Глазнев Виктор Николаевич, заведуюший кафедрой геофизики, доктор физико-математических наук

E-mail: glaznev@geol.vsu.ru

Тел.: 84732208385

АО Предприятие подводно-технических работ «Пётр», 2. Воронеж

Стариков Вадим Сергеевич, инженер - геофизик

E-mail:stvase@mail.ru

Тел.: +79204005720, +79103461130
26. Николаев, Г. А. Сварка в машиностроении: Справочник в 4-х т. / Г. А. Николаев. - М.: Машиностроение. - 1978 (1-4 T).

27. Analyses of magnetic field in spiral steel pipe / Y. Zhang [et al.] // Journal of Magnetism and Magnetic Materials, 2015. 375. - 1. - P. 210-216.

28. Some remarks on static field thin sheet models / L. Eskola [et al.] // Journal of Applied Geophysics, 1993. - 30. - P. 229234.

29. Нефтегазовые трубные системы [Электронный ресурс] www.gkr-ngts.ru/informaciya/tehnologii-proizvodstva-stalnyhtrub (дата обращения 19.07.2018).

30. Furness, $P$. The magnetic fields of steel drums / P. Furness // Journal of Applied Geophysics, 2002. - 51. - P. 63-74.

31. Кирьянов, Д. В. Mathcad14 / Д. В. Кирьянов. - СПб.: БХВ-Петербург. - 2007. - 704 с.

Voronezh State University

Glaznev V. N., Head of the Chair, Doctor of Physical and Mathematical Sciences

E-mail: glaznev@geol.vsu.ru

Tel.: 8(473) 220-8385

«PETER» JOINT - STOCK C ${ }^{\circ}$. Diving Services, Voronezh

Starikov V. S., engineer - geophysicist

E-mail:stvase@mail.ru

Tel.: +79204005720, +79103461130 